\title{
Jerk analysis of a module of an artificial spine by means of screw theory
}

\author{
J. Gallardo-Alvarado*, R. Lesso-Arroyo \\ Department of Mechanical Engineering, INSTITUTO TECNOLÓGICO DE CELAYA \\ Av. Tecnológico y A. García Cubas, 38010 Celaya, GTO, México \\ *gjaime,rlesso@itc.mx
}

\begin{abstract}
In this work, a novel parallel manipulator is introduced with the purpose of simulating the jerk analysis of the end of the spine. The displacement analysis is presented in a semi-closed form solution whereas the velocity, acceleration and jerk analyses are carried out by means of the theory of screws.

Keywords: Parallel manipulator, spine, screw theory, jerk analysis, kinematics.

\section{RESUMEN}

En este trabajo se introduce un manipulador paralelo novedoso con el propósito de simular el análisis de pulso de la columna vertebral. El análisis de posición se presenta en forma semi cerrada, mientras que los análisis de velocidad, aceleración y pulso son realizados por medio de la teoría de tornillos.

Palabras clave: Predicción lineal, servo control visual, seguimiento, visión estéreo, calibración de cámaras.
\end{abstract}

\section{Introduction}

The jerk, a research field of great interest in quite different academic communities, is the time rate of change acceleration and it is related to the rate of change of force, namely, an impulse which is considered as a hammer blow force. Particularly, it is known that there is a direct relationship between the jerk and the movements of the human body $[1,2,3]$. Consider, for instance, that Crossman and Goodeve [4] have shown that when high spatial precision is required, many movements related with the hands present irregularities and multiple velocity peaks. Gielen et al. [5] noted that the charactecteristic pattern of cerebellar ataxia, related with the jerk and submovements, is contained in the trajectory of the hand during repeated arm movements. Goldvasser et al. [6] investigated the high curvature analysis and the integrated absolute jerk for differentiating healthy and cerebellopathy patients performing pointing tasks. In order to understand how the central nervous system controls the kinematics of rapid finger and hand movement, Novak et al. [7] proposed an objective algorithm to identify overlapping submovements, detecting appreciable inflections in the acceleration traces which are related, evidently, with the jerk. Viviani and coworkers $[8,9]$ proved that there is a correlation between perception, motion planning, and jerk in human. The spinal column has been the subject of an exhaustive field research.

Basically, the motions of the spine can be classified in three types: a) sagittal plane movement, b) coronal plane movement and c) transversal plane movement of axial rotation. The individual segmental range of motions was quantified for all spinal levels by Panjabi and White [10]. Dimnet et al. [11] reported a technique, based on lateralview-X-ray, to determine parameters for describing the centers of rotation and curvature of the spine. Gracovetsky and Farfan [12] proposed a novel theory based on the mechanical behavior of intervertebral joints capable of computing both spinal motions and muscular actions. By means of the technique of videofluoroscopy, Cholewicki and 
McGill [13] studied the kinematics of the lumbar spine. Yoganandan [14] determined the kinematic response of the lumbar spine using instrumented transpedicular screws and plates. It is well-known that a structure is any assemblage of materials that is intended to sustain loads, a strong argument to simulate the spine, in that way Levin [15] identifies tensegrity structures whose elements always work in tension regardless of the direction of the applied force, an interesting option to simulate the kinematics of the spine. Willems et al. [16] provided preliminary information about the spatial kinematics of the thoracic spine in vivo. Faber et al. [17] proposed a method to compute Euler's angles of rotation of a body segment during locomotion and applied it to in-vivo spinal kinematics. In order to demonstrate in-vivo intervertebral coupled motions of the upper cervical spine, Yoshikawa et al. [18] studied the spatial kinematics of the upper cervical spine during head rotation using threedimensional magnetic resonance imaging (MRI) in healthy volunteers. Ziddiqui et al. [19] investigated the in-vivo sagittal kinematics of the lumbar spine at the instrumented level. Ishii et al. [20] studied the spatial kinematics of the cervical spine during lateral bending while Konz et al. [21] investigated the spatial kinematics of spinal during walking. Chanceya et al. [22] determined the center of rotation of the upper cervical considering pure bending. Gill et al. [23] examined the effect of changes in horizontal lift distance on the amount of flexion in different spine regions according to different lift styles. In order to approach the socalled shaken baby syndrome, recently, Jones et al, [24] proposed a methodology for the kinematic analysis of infant spine.

It is straightforward to show that the movements of the spinal column can be simulated by assembling several parallel manipulators in series connection. In this way, in a recent contribution, Zhu et al. [25] proposed some parallel manipulators to simulate the finite kinematics of the end of the spinal column. In this work, not only the finite kinematics but also the velocity, acceleration and jerk analyses of the end of the spine are carried out by applying the theory of screws to a novel parallel manipulator.

\section{Description of the parallel manipulator}

The proposed parallel manipulator, see Fig. 1, consists of a moving platform and a fixed platform connected to each other by means of three Universal + Prismatic + Spherical (UPS-type) limbs and two Revolute + Revolute + Revolute + Spherical (RRRS-type) limbs, where the active kinematic pairs are indicated with underlines.

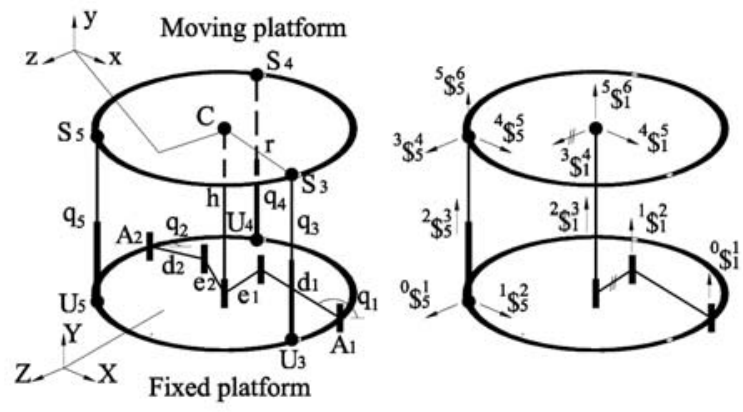

Figure 1. The proposed base parallel manipulator

According to a revised version of the KutzbachGrübler formula, this parallel manipulator possesses five degrees of freedom, three rotations that are provided by the translational generalized coordinates $\{q 3, q 4, q 5\}$ and two translations that are provided by the rotational generalized coordinates $\{q 1, q 2\}$. The main merit of this topology, for brevity's sake: 3R2T, is that the position and orientation of the moving platform with respect to the fixed platform are controlled independently by means of the UPS-type limbs and the RRRS-type limbs, respectively; therefore, this spatial mechanism belongs to the class known as Decoupled parallel manipulators. 


\section{Finite Kinematics}

In this section, the displacement analysis of the mechanism under study is presented. The inverse position analysis (IPA) consists of finding the generalized coordinates of the parallel manipulator given the pose, position and orientation of the moving platform with respect to the fixed platform whereas the forward position analysis (FPA) consists of finding the pose of the moving platform given the generalized coordinates of the parallel manipulator.

Let $X Y Z$ be a global reference frame attached to the fixed platform and let xyz be a reference frame attached to the moving platform, see Fig. 1. Given the position, vector $C$ associated to point $C$, and orientation, rotation matrix $\mathrm{R}$ of the moving platform with respect to the fixed platform, angles $q 1$ and $q 2$ are obtained directly from the closure equations:

$A_{i}+d_{i}+e_{i}+h=C \quad i=1,2$

On the other hand, lengths $q 3, q 4$ and $q 5$ are obtained as follows:

$q_{i}^{2}=\left(S_{i}-U_{i}\right) \cdot\left(S_{i}-U_{i}\right) \quad i=3,4,5$

where the dot - denotes the usual dot product of the three dimensional vectorial algebra whereas

$\mathbf{S}_{i}$ and $\mathbf{U}_{i}$ denote, respectively, the position vector

of points $\mathrm{S}_{i}$ and $\mathrm{U}_{i}$. Furthermore, any point $\mathrm{P}$ is computed upon point $p$, which is expressed in the frame $x y z$, as follows:

$\left[\begin{array}{c}P \\ 1\end{array}\right]=\left[\begin{array}{cc}R & C \\ 0_{1 \times 3} & 1\end{array}\right]\left[\begin{array}{l}p \\ 1\end{array}\right]$

In order to compute the FPA, point $C$ is found rewriting Eq. (1) as

$$
\begin{aligned}
\left(C-A-d_{i}-h\right) \cdot\left(C-A-d_{i}-h\right)= & e_{i}^{2} \\
& i=1,2 \quad(4)
\end{aligned}
$$

which yields a closed-form solution. Furthermore, with the purpose of computing lengths $q 3, q 4$ and $q 5$, let us consider the following closure equations:

$$
\left.\begin{array}{l}
\left(S_{i}-U_{i}\right) \cdot\left(S_{i}-U_{i}\right)=q_{i}^{2} \quad i=3,4,5 \\
\left(S_{i}-C\right) \cdot\left(S_{i}-C\right)=r^{2} \\
\left(S_{3}+S_{4}+S_{5}\right) / 3=C
\end{array}\right\}
$$

where, $r$ is the radius of the moving platform. Expressions (5) are solved using recursively the Sylvester dialytic elimination method, for details, the reader is referred to $[26,27,28]$, yielding a semi-closed form solution. With this procedure, sixteen possible locations, including reflected solutions, of the moving platform with respect to the fixed platform are available. Furthermore, considering that two different locations are available for point $C$, then the moving platform can reach 32 different locations or poses with respect to the fixed platform. Once the coordinates of points $S_{i}(\mathrm{i}=3,4,5)$ are computed, rotation matrix $\mathrm{R}$ is obtained by applying the method introduced in Gallardo-Alvarado et al. [29].

\section{Infinitesimal kinematics}

The mathematical tool to approach the velocity, acceleration and jerk analyses is the theory of screws.

Let $\mathbf{V}_{p}=\left[\omega ; \mathbf{V}_{p}\right]^{\top}$ be the velocity state, or twist about a screw [30], of the moving platform with respect to the fixed platform, where $\omega$ and $\mathbf{v}_{\mathrm{p}}$ are, respectively, the angular and linear velocities of the moving platform with respect to the fixed platform so that subscript $P$ denotes a point of the moving platform that is instantaneously coincident with a point fixed at the global reference frame, usually such point is the origin of the global 
reference frame. Furthermore, the velocity state can be written in screw form, the infinitesimal screws of two representative legs are depicted in Fig. 1, through any of the limbs as follows

$J_{i} \Omega_{i}=V p \quad i=1,2, \ldots, 5$

Where, $\mathrm{J}_{i}=\left[\begin{array}{llllll}{ }^{0} \$_{i}^{1} & { }^{1} \$_{i}^{2} & { }^{2} \$_{i}^{3} & { }^{3} \$_{i}^{4} & { }^{4} \$_{i}^{5} & { }^{5} \$_{i}^{6}\end{array}\right]$

is the screw-coordinate Jacobian matrix of the i-th limb and

$$
\Omega_{i}=\left[{ }_{0} \omega_{1}^{i} ;_{1} \omega_{2}^{i} ;_{2} \omega_{3}^{i} ;_{3} \omega_{4}^{i} ;_{4} \omega_{5}^{i} ;_{5} \omega_{6}^{i}\right]
$$

is a matrix containing the joint velocity rates of the i-th leg. It is important to mention that joint velocity rates

$$
\left\{{ }_{0} \omega_{1}^{1}=q_{1} ;_{0} \omega_{1}^{2}=q_{2} ;_{2} \omega_{3}^{3}=q_{3} ; \omega_{3}^{4}=q_{4} ; \omega_{0} \omega_{1}^{5}=q_{5}\right\}
$$

have the privilege to be considered as the active kinematic pairs of the base module.

Furthermore, it must be noted that screws ${ }^{2} \$_{i}^{3}(i=1,2)$ are elements related to fictitious prismatic pairs in which evidently ${ }_{2} \omega_{3}^{1}={ }_{2} \omega_{3}^{2}=0$. These fictitious elements are included only for the sake of completing an algebraic requirement, specifically with the purpose of completing the rank of the Jacobian matrix of limbs 1 and 2 .

The inverse velocity analysis (IVA), a necessary task to approach the acceleration analysis, consists of finding the joint velocity rates of the limbs given the velocity state $V_{p}$. The IVA is computed directly from Eq. (6). On the other hand, the forward velocity analysis (FVA) consists of finding the velocity state $V_{P}$ given a set of active joint velocity rates

$$
\left\{q_{1}, q_{2}, q_{3}, q_{4}, q_{5}\right\}
$$

The FVA is simplified considerably by applying the concept of reciprocal screw. To this end, please note that screw ${ }^{3} \$_{1}^{4}$ is reciprocal to all the screws of limb 1 , excepting screw ${ }^{0} \$_{1}^{1}$ which is associated to the active joint velocity rate $\dot{q}_{1}$ Therefore, the application of the Klein form of screw ${ }^{3} \$_{1}^{4}$ with both sides of the corresponding Eq. (6), the reduction of terms leads to

$\left\{{ }^{3} \$_{1}^{4} ; V_{p}\right\}=\dot{q}_{1}\left\{{ }^{3} \$_{1}^{4} ;{ }^{0} \$_{1}^{1}\right\}$

similarly, from limb 2 it follows that

$\left\{{ }^{3} \$_{2}^{4} ; v_{p}\right\}=\dot{q}_{2}\left\{{ }^{3} \$_{2}^{4} ;{ }^{0} \$_{2}^{1}\right\}$

Furthermore, screws ${ }^{5} \$_{4}^{6} ; \$_{5}^{6}$ and ${ }^{5} \$_{6}^{6}$ are reciprocal to all the revolute joints in the same limb.

Therefore,

$\left\{{ }^{5} \$_{i}^{6} ; V_{p}\right\}=\dot{q}_{i} \quad i=4,5,6$

In order to satisfy an algebraic requirement, note that screw ${ }^{5} \$_{1}^{6}$ is reciprocal to all the screws of $\operatorname{limb} 1$, therefore, one can obtain

$\left\{{ }^{5} \$_{1}^{6} ; V_{p}\right\}=0$

Casting into a matrix-vector form Eqs. (7)-(10), a compact velocity expression is formulated as follows:

$J^{T} \Delta V_{p}=Q_{v e l}$

Where, $J=\left[{ }^{3} \mathbb{S}_{1}^{4} ; \quad{ }^{3} \mathbb{S}_{2}^{4} ; \quad{ }^{5} \mathbb{S}_{3}^{6} ; \quad{ }^{5} \mathbb{S}_{4}^{6} ; \quad{ }^{5} \mathbb{S}_{5}^{6} ; \quad{ }^{5} \mathbb{S}_{1}^{6}\right]$ is the active screw-coordinate Jacobian matrix, 
$\Delta=\left[\begin{array}{cc}0_{3 \times 3} & \mathrm{I}_{3} \\ \mathrm{I}_{3} & 0_{3 \times 3}\end{array}\right]$ is an operator of polarity, and

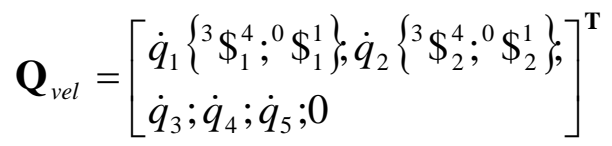

is a matrix containing the generalized speeds of the base module. Hencefore, velocity state $V_{P}$ is obtained directly from expression (11).

In what follows, the acceleration analysis is approached using the same methodology used for the velocity analysis.

Let $\mathbf{A}_{p}=\left[\dot{\omega}, \mathbf{a}_{p}-\omega \times \mathbf{v}_{p}\right]^{\mathbf{T}}$ be the reduced acceleration state, or accelerator for brevity's sake, of the moving platform with respect to the fixed platform, where $\dot{\omega}$ and $a_{p}$ are, respectively, the angular and linear accelerations of the moving platform with respect to the fixed platform, taking point $P$ as the reference pole. Then, the accelerator can be written in screw form, see Rico and Duffy [31], through any of the limbs as follows:

$J_{i} \dot{\Omega}_{i}+A_{i}=A_{p} \quad i=1,2, \ldots, 5$

Where,

$A_{i}=\Sigma_{j=0}^{4}\left[{ }_{j} \omega_{j+1}^{j} \quad{ }^{j} \$_{i}^{j+1} \quad \sum_{k=j+1}^{5} \quad{ }_{k} \omega_{k+1}^{j} \quad{ }^{k} \$_{i}^{k+1}\right]^{T}$

is the acceleration screw of the i-th limb in which the brackets $[* *$ denotes the Lie product, and $\dot{\Omega}_{\boldsymbol{q}}$ is a matrix containing the time derivatives of the elements of matrix $\Omega_{1}$.

The inverse acceleration analysis (IAA) consists of finding the joint acceleration rates of the limbs of the parallel manipulator given the accelerator

$A_{\rho}$ and is computed directly from expression (12). On the other hand, the forward acceleration analysis (FAA) consists of finding the reduced acceleration state $A_{p}$ given a set of active joint acceleration rates $\left\{\ddot{q}_{1}, \ddot{q}_{2}, \ddot{q}_{3}, \ddot{q}_{4}, \ddot{q}_{5}\right\}$. Following the trend of the FVA, the systematic application of the Klein form between the reciprocal screws indicated in the FVA with both sides of expression(12), and casting into a matrix-vector form the equations, thus, derived yields the following expression:

$J^{T} \Delta A_{p}=Q_{a c c e l}$

where,

$$
Q_{\text {accel }}=\left[\begin{array}{c}
\ddot{q}_{1}\left\{{ }^{3} \$_{1}^{4} ; 0{ }^{1}\right\}+\left\{{ }^{3} \$_{1}^{4} ; A\right\} \\
\ddot{q}_{2}\left\{{ }^{3}{ }^{4}{ }_{2}^{4} ;{ }^{0}{ }_{2}^{1}\right\}+\left\{{ }^{3} \$_{2}^{4} ; A_{2}\right\} \\
\ddot{q}_{3}+\left\{{ }^{5} \$_{3}^{6} ; A_{3}\right\} \\
\ddot{q}_{4}+\left\{{ }^{5} \$_{4}^{6} ; A_{4}\right\} \\
\ddot{q}_{5}+\left\{{ }^{5} \$_{5}^{6} ; A_{5}\right\} \\
\left\{{ }^{5}{ }_{1}^{6} ; A_{1}\right\}
\end{array}\right]
$$

Therefore, the reduced acceleration state $A_{p}$ is obtained directly from expression (13).

This section finishes with the main purpose of this contribution, the jerk analysis of the artificial spine.

Let $\mathbf{J}_{p}=\left[\ddot{\omega}, \mathbf{j}_{p}-2 \dot{\omega} \times \mathbf{v}_{p}-\omega \times \mathbf{a}_{p}\right]^{\mathrm{T}}$ be the reduced jerk state or jerkor, for brevity's sake, of the moving platform with respect to the fixed platform, where $\ddot{\omega}$ and jP are, respectively, the angular and linear jerks of the moving platform with respect to the fixed platform, taking point $P$ as the reference pole.

Furthermore, the jerkor can be written in screw form, see Gallardo-Alvarado et al. [32], through any of the limbs as follows:

$J_{i} \ddot{\Omega}_{i}+\Gamma_{i}=J_{p} \quad i=1,2, \ldots, 5$

Where, 


$$
\begin{aligned}
\Gamma_{i} & =2 \Sigma_{j=0}^{4}\left[\begin{array}{lll}
{ }^{j} V^{j+1} & \sum_{k=j+1}^{5} & { }^{k} A^{k+1}
\end{array}\right] \\
& +\Sigma_{j=0}^{4}\left[\begin{array}{lll}
{ }^{j} A^{j+1} & \sum_{k=j+1}^{5} & { }^{k} V^{k+1}
\end{array}\right] \\
& +\Sigma_{j=0}^{4}\left[\begin{array}{llll}
{ }^{j} V^{j+1} & {\left[{ }^{j} V^{j+1}\right.} & \sum_{k=j+1}^{5} & { }^{k} V^{k+1}
\end{array}\right]
\end{aligned}
$$

is the jerk screw of the i-th limb and $\ddot{\Omega}_{1}$ is a matrix containing the time derivatives of the elements of matrix $\ddot{\Omega}$.

The inverse jerk analysis (IJA) consists of finding the joint jerk rates of the parallel manipulator given jerkor $\mathrm{J}_{P}$ and is computed directly from expression (15). On the other hand, the forward jerk analysis (FJA) consists of finding jerkor $J_{P}$ given a set of active joint jerk rates $\left\{\dot{\ddot{q}}_{1}, \dot{\ddot{q}}_{2}, \dot{\ddot{q}}_{3}, \dot{\ddot{q}}_{4}, \dot{\ddot{q}}_{5}\right\}$ Following the trend of the FVA and FAA, the systematic application of the Klein form between the reciprocal screws indicated in the FVA with both sides of expression (15), and casting into a matrix-vector form the equations thus derived, yields the following expression:

$$
J^{T} \Delta J_{p}=Q_{j e r k}
$$

Where,

$$
Q_{j e r k}=\left[\begin{array}{c}
\dot{\ddot{q}}_{1}\left\{{ }^{3} \$_{1}^{4} ; 0 \$_{1}^{1}\right\}+\left\{{ }^{3} \$_{1}^{4} ; \Gamma_{1}\right\} \\
\dot{\ddot{q}}_{2}\left\{{ }^{3} \$_{2}^{4} ;{ }^{0} \$_{2}^{1}\right\}+\left\{{ }^{3} \$_{2}^{4} ; \Gamma_{2}\right\} \\
\dot{\ddot{q}}_{3}+\left\{{ }^{5} \$_{3}^{6} ; \Gamma_{3}\right\} \\
\dot{\ddot{q}}_{4}+\left\{{ }^{5} \$_{4}^{6} ; \Gamma_{4}\right\} \\
\dot{\ddot{q}}_{5}+\left\{{ }^{5} \$_{5}^{6} ; \Gamma_{5}\right\} \\
\left\{{ }^{5}{ }_{1}^{6} ; \Gamma_{1}\right\}
\end{array}\right]
$$

Therefore, reduced jerk state JP is obtained directly from expression (16).

Finally, once the velocity, reduced acceleration and reduced jerk states are computed using the origin of the global reference frame $X Y Z$ as the pole, the linear and angular properties of any point attached to the moving platform, for example its geometric centre, are computed by applying the concept of helicoidal vector fields, for details see GallardoAlvarado et al. [32].

\section{Numerical example}

The parameters, using hereafter SI units, and generalized coordinates of the numerical example are listed in Table 1.

$$
\begin{aligned}
& A_{1}=(0.03,0,-0.051961) \\
& A_{2}=(0.03,0,0.051962) \\
& U_{3}=(0.06,0,0) \\
& U_{4}=(-0.03,0,-0.051961) \\
& U_{5}=(-0.03,0,0.051961) \\
& h=0.08, r=0.0 .08246 \\
& q_{1}=3.403+0.174 \sin (t) \cos (t) \\
& q_{2}=1.309+0.0872 \sin (t) \\
& q_{3}=0.08+0.005 \sin (t) \\
& q_{4}=0.085+0.015 \sin (t) \\
& q_{5}=0.0825+0.01 \sin (t) \sin (t)
\end{aligned}
$$

Table 1. Parameters of the numerical example

With these data the resulting time history of the angular and linear jerks of the moving platform, with respect to the fixed platform, is provided in Fig. 2. 

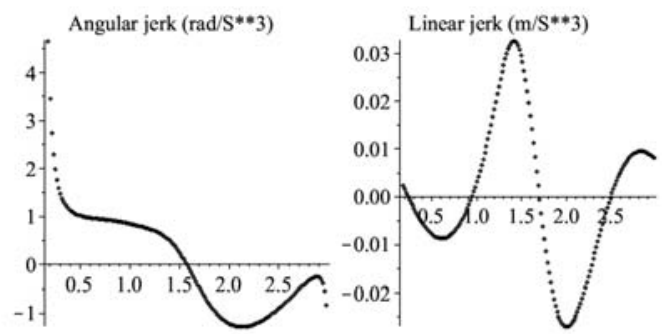

$\mathrm{X}$ axis

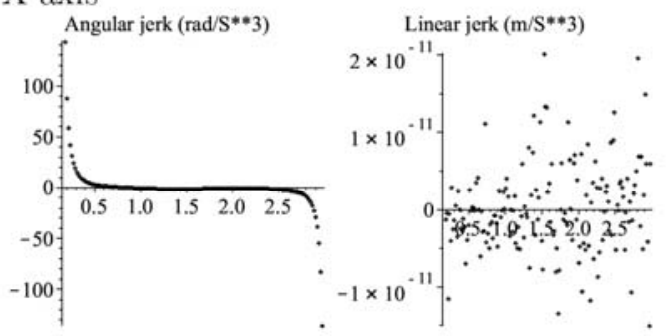

$\mathrm{Y}$ axis

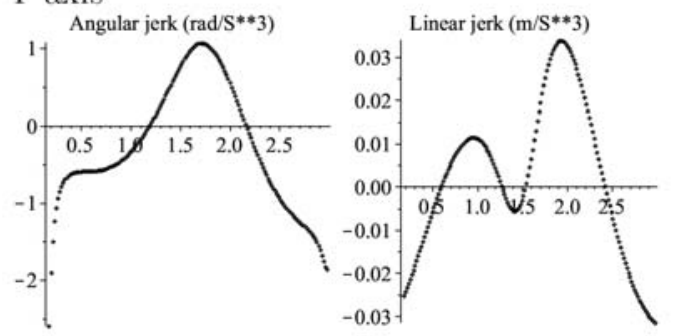

$\mathrm{Z}$ axis

Figure 2. Time history of the forward jerk analysis of the centre of the moving platform.

Please note that, as it was expected, the linear jerk along the $Y$ axis is practically null. Furthermore, in order to avoid singular configurations, the considered interval for time $t$ was

$0<\mathrm{t}(\mathrm{S})<\pi$.

\section{Discussion}

In this work, a novel five-degrees-of-freedom parallel manipulator is proposed for simulating the jerk analysis of a module of the spine. This manipulator brings the following features:

- Decoupled architecture, which is a combination of a spherical parallel manipulator with a planar parallel manipulator for controlling, respectively, the orientation and position of the moving platform with respect to the fixed platform.

- Due to the decoupled topology, the forward displacement analysis is presented in a semi-closed form solution. All the feasible locations of a platform with respect to the other can be calculated given the five generalized coordinates.

- Simple and compact expressions for solving the velocity, acceleration and jerk analyses are derived here by applying the concept of reciprocal screws via the Klein form of the Lie algebra $e(3)$ which is isomorphic to the theory of screws.

- The proposed parallel manipulator is a nonoverconstrained parallel manipulator and, unlike the parallel manipulators introduced in Zhu et al., [25] does not require additional conditions of manufacture.

Finally, a numerical example is provided.

Conflicts of interest

None

\section{References}

[1] Morasso, P. Spatial control arm movements. Exp. Brain Res., Vol. 42, pp. 223-227 (1981).

[2] Flash, T. and Hogan, N. The coordination of arm movements: an experimentally confirmed mathematical model. J. Neurosci., Vol. 5, pp. 1688-1703 (1985).

[3] Uno, Y., Kawato, M., and Suzuki, R. Formation and control of optimal trajectory in human multijoint arm movements. Biol. Cybern., Vol. 61, pp. 89-101 (1989).

[4] Crossman, E. R. F.W. and Goodeve, P. J. Feedback control of handmovements and Fitts law. Q. J. Exp. Psychol., Vol. A35, pp. 251-278 (1983). 
[5] Gielen, C. C., Vrijenhock, E. J., and Neggers, S. F. Arm position constraints during pointing and reaching in 3-D space. J. Neurophysiol., Vol. 78, pp. 660-673 (1997).

[6] Goldvasser, D., McGibbon, C. A., and Krebs, D. E. High curvature and jerk analyses of arm ataxia. Biol. Cybern., Vol. 84, pp. 85-90 (2001).

[7] Novàk, K.E.,Miller,L.E.,and Houk,J. C.Kinematic properties of rapid hand movements in a knob turning task. Exp. Brain Res., Vol. 132, pp. 419-433 (2000).

[8] Viviani, P. and Schneider, R. A. development study of the relationship between geometry and kinematics in drawing movements. J. Exp. Psychol. Hum. Percept. Perform., Vol. 17, pp. 198-218 (1991).

[9] Viviani, P. and Flash, T. Minimum-jerk, two-thirds power law, and isochrony: converging approaches tomovement planning. J. Exp. Psychol. Hum. Percept. Perform., Vol. 21, pp. 32-53 (1995).

[10] Panjabi, M.M., White, A.A. Basic biomechanics of the spine. Neurosurgery, Vol. 7, pp. 76-93 (1980).

[11] Dimnet, J., Pasquet, A., Krag, M.H., Panjabi, M.M. Cervical spine motion in the sagittal plane: Kinematic and geometric parameters. Journal of Biomechanics, Vol. 15, pp. 959-969 (1982).

[12] Gracovetsky, S., Farfan, H. The optimum spine. Spine, Vol. 11, pp. 543 (1986).

[13] Cholewicki, J., McGill, S.M. Lumbar spine kinematics obtained from videofluoroscopy. Journal of Biomechanics, Vol. 25, pp. 801 (1992).

[14] Yoganandan, N., Pintar, F., Maiman, D. J., Reinartz, J., Sances, A., Larson, S.J., Cusick, J.F. Kinematics of the lumbar spine following pedicle screw plate fixation. Spine, Vol. 18, pp. 504-512 (1993).

[15] Levin, S.M. The importance of soft tissue for structural support of the body. In Dorman, T.A., editor. Prolotherapy in the lumbar spine and pelvis, Spine: State of the art reviews, Vol. 9, pp. 357 (1995).
[16] Willems J.M., Jull G.A., Ng, J.K.-F. An in vivo study of the primary and coupled rotations of the thoracic spine. Clinical Biomechanics, Vol. 11, pp. 311-316 (1996).

[17] Faber, M.J., Schamhardt, H.C., van Weeren, P.R. Determination of 3D spinal kinematics without defining a local vertebral coordinate system. Journal of Biomechanics, Vol. 32, pp. 1355-1358 (1999).

[18] Yoshikawa, H., Ishii, T., Mukai, Y., Hosono, N., Sakaura, H., Nakajima, Y., Sato, Y., Sugamoto, K. Kinematics of the upper cervical spine in rotation: In vivo three-dimensional analysis. Spine, Vol. 29, pp. E139-E144 (2004).

[19] Ziddiqui, M., Karadimas, E., Nicol, M., Smith, F.W., Wardlaw, D. Effects of X-stop device on sagittal lumbar spine kinematics in spinal stenosis. Journal of Spinal Disorden Technology, Vol. 19, pp. 328-333 (2006).

[20] Ishii, T., Mukai, Y., Hosono, N., Sakaura, H., Fujii, R., Nakajima, Y., Tamura, S., Iwasaki, M., Yoshikawa, H., Sugamoto, K. Kinematics of the cervical spine in lateral bending: In vivo three-dimensional analysis. Spine, Vol. 31, pp. 155-160 (2006).

[21] Konz, R.J., Fatone, S., Stine, R.L., Ganju, A., Gard, S.A., Ondra, S.L. A kinematic model to assess spinal motion during walking. Spine, Vol. 31, pp. E898-E906 (2006).

[22] Chanceya, V.C., Ottaviano, D., Myers, B.S., Nightingale, R.W. A kinematic and anthropometric study of the upper cervical spine and the occipital condyles. Journal of Biomechanics, Vol. 40, pp. 1953-1959 (2007).

[23] Gill, K.P., Bennett, S.J., Savelsbergh, G.J.P., van Dieën, J.H. Spine, Vol. 32, pp. 1599-1604 (2007).

[24] Jones, M., Holt, C., Franyuti, D. Developing a methodology for the analysis of infant spine kinematics for the investigation of the shaken baby syndrome. Journal of Biomechanics, Vol. 41, pp. S3-55 (2008). 
[25] Zhu, S.J., Huang, Z., Zhao, M.Y. Feasible HumanSpine Motion Simulators Based on Parallel Manipulators Source: Parallel Manipulators, Towards New Applications, Book edited by: Huapeng Wu, ISBN 978-3902613-40-0, pp. 506, I-Tech Education and Publishing, Vienna, Austria (2008).

[26] Innocenti, C, Parenti-Castelli, V. Direct position analysis of the Stewart platform mechanism. Mechanism and Machine Theory, Vol. 35, pp. 611-621 (1990).

[27] Tsai, L.-W. Robot analysis, John Wiley \& Sons, (1999).

[28] Gallardo-Alvarado, J., Rodríguez-Castro, R., Nazrul Islam, Md. Analytical solution of the forward position analysis of parallel manipulators that generate 3-RS structures. Advanced Robotics, Vol. 22, pp. 215-234 (2008a).

[29] Gallardo-Alvarado, J., Aguilar-Nájera, C.R., CasiqueRosas, L., Pérez- González, L., Rico-Martínez, J.M. Solving the kinematics and dynamics of a modular spatial hyper-redundant manipulator by means of screw theory. Multibody System Dynamics, Vol. 20, pp. 307325 (2008b).

[30] Ball, R.S. A Treatise on the Theory of Screws, Cambridge University Press: Cambridge. 1900 (reprinted 1998).

[31] Rico, J.M., Duffy, J. An Application of screw algebra to the acceleration analysis of serial chains. Mechanism and Machine Theory, Vol. 31, pp. 445-457 (1996).

[31] Rico, J.M., Duffy, J. An Application of screw algebra to the acceleration analysis of serial chains. Mechanism and Machine Theory, Vol. 31, pp. 445-457 (1996).

[32] Gallardo-Alvarado, J., Orozco-Mendoza, H., Rodríguez-Castro, R. Finding the jerk properties of multibody systems using helicoidal vector fields. Proceedings of the Institution of Mechanical Engineers, Part C: Journal of Mechanical Engineering Science, Vol. 222, pp. 2217-2229 (2008c). 


\section{Authors' Biography}

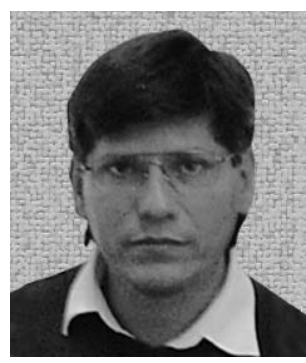

\section{Jaime GALLARDO-ALVARADO}

He received the M.Sc. in mechanical engineering in 1989 from the Instituto Tecnológico de Celaya, Mexico, and the Ph.D. in electrical engineering in 1999 from the Instituto Tecnológico de la Laguna, Mexico. For several years, Prof. Gallardo worked at prestigious research institutions founded by the National Council of Science and Technology of Mexico, CONACYT, such as IMEC and CIATEQ. In 1993, he joined the Department of Mechanical Engineering of the Instituto Tecnológico de Celaya where, currently, he is a full-time professor. He is a member of the National System of Researchers in Mexico, SNI. His research fields include spatial kinematics and dynamics, more specifically, kinematics and dynamics of robotic systems using the screw theory, development of software engineering and machine theory. Prof. Gallardo has authored/co-authored more than 30 regular papers approaching these topics published in several journals.

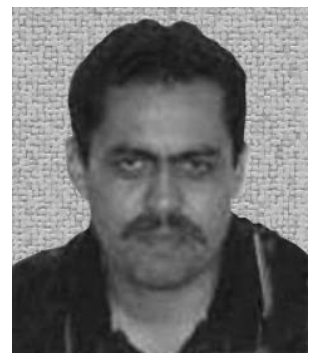

\section{Raúl LESSO-ARROYO}

He received his bachelor's degree in mechanical engineering in 1991, the M.Eng. in mechanical engineering in 1993, both from the Universidad de Guanajuato, Mexico, and the M.Sc. in finite element method from the Universidad de Educación a Distancia UNED, Spain. For many years, Prof. Lesso worked for different companies and prestigious research institutions in Mexico. In 1994, he joined the Department of Mechanical Engineering of the Instituto Tecnológico de Celaya where, currently, he is a full-time professor. His main interests include finite element method, biomechanics and computer aided design. 